\title{
Effects of secukinumab on serum adipocytokines: preliminary data
}

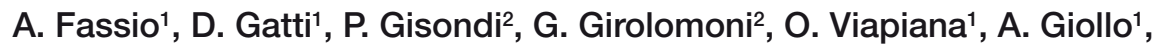 \\ M. Zamboni ${ }^{3}$, M. Rossini' ${ }^{1}$, L. Idolazzi ${ }^{1}$ \\ ${ }^{1}$ Unit of Rheumatology, University of Verona, Italy; ${ }^{2}$ Unit of Dermatology, University of Verona, Italy; \\ ${ }^{3}$ Unit of Geriatrics, University of Verona, Italy
}

\begin{abstract}
SUMMARY
Psoriatic arthritis (PsA) is a chronic inflammatory disease that affects joints, connective tissues and the axial skeleton. Metabolic syndrome is an independent risk factor for psoriasis (Pso) development and is associated with more severe forms of Pso. Adipocytokines are secreted by white adipose tissue and are thought to link obesity with the development of metabolic and cardiovascular diseases. Secukinumab is a new monoclonal antibody with a different mechanism of action. This antibody selectively binds to and neutralizes interleukin-17 (IL-17) and it has shown efficacy in the treatment of PsA. The aim of this study was to evaluate the possible interferences of secukinumab on different adipocytokines.

We enrolled 28 patients with PsA, classified with the CASPAR criteria. Serum samples were stored at baseline and then at the first, the third and the sixth month of therapy. Resistin, chemerin, adiponectin and C-reactive protein (CRP) were dosed.

When tested globally, none of the adipokine tested showed any statistically significant variation. However, when the male group was tested, both resistin and chemerin at M6 showed a significant decrease from baseline. CRP did not show any variation at any time point.

Our study demonstrated that treatment with secukinumab has little influence on the levels of adipokines tested within the first six months of treatment even though it might exert different influence between males and females from a metabolic perspective. Further studies with greater numbers of patients are needed to determine whether these preliminary results have clinical relevance.
\end{abstract}

Key words: Psoriasis; psoriatic arthritis; secukinumab; adipokines; resistin; adiponectin; chemerin.

\section{INTRODUCTION}

Doriasis (Pso) is a common chronic inflammatory disease involving the skin that can be associated with several comorbidities, one of which is psoriatic arthritis (PsA) (1). Psoriatic arthritis is a chronic, systemic inflammatory disease affecting peripheral joints, connective tissues, and the axial skeleton and is associated with psoriasis of the skin and nails (1).

Patients with Pso and PsA share obesity (and related complications) and cardiovascular (CV) diseases. Chronic inflammation in Pso and PsA can partially explain the development of atherosclerosis and $\mathrm{CV}$ diseases, but also the concomitant alterations in the glucose metabolism (diabetes and insulin resistance) and lipids (dyslipidaemia) enhance this risk (2). Systemic inflammation induced by obesity and metabolic syndrome could exacerbate local inflammation, worsening both Pso and PsA (3). Indeed, increasing evidence is suggesting that metabolic syndrome is an independent risk factor for Pso development and is associated with more severe forms of Pso (3).

Nowadays, adipose tissue is considered as an active endocrine and immune organ, producing various cytokines, named adipocytokines, and they link together inflammation and metabolic disorders (4). Adipocytokines, in cooperation with macrophages and $\mathrm{T}$ cells, induce and modulate adipose tissue inflammation, contribute to the pathogenesis of metabolic syndrome and enhance local inflammation at skin and
Corresponding author Angelo Fassio Unit of Rheumatology University of Verona

Ospedale Civile Maggiore, Verona, Italy E-mail: angelo.fassio@yahoo.it 
articular level. Adipocytokines are secreted by white adipose tissue and are thought to link obesity with the development of metabolic diseases (5).

Many adipocytokines, including adiponectin, resistin and chemerin contribute to the development of insulin resistance and lowgrade inflammation $(6,7)$. Adiponectin and resistin exert opposite effects on glucose metabolism: while adiponectin sensitises tissues to insulin, the latter promotes development of insulin resistance (8). Chemerin is also an adipocytokine implicated in the immune response with both pro and antiinflammatory properties (6). All these cytokines not only play roles in metabolism but also are involved in development of chronic inflammation (9).

Inhibitors of tumor necrosis factor (TNFi) have significantly improved outcomes among patients with psoriatic arthritis and have been available for the treatment of PsA for many years now (10). On the contrary, secukinumab is a new monoclonal antibody with a different mechanism of action. This antibody selectively binds to and neutralizes interleukin-17 (IL-17) and it has shown efficacy in the treatment of psoriasis, ankylosing spondylitis and psoriatic arthritis (11). IL-17 is an important cytokine involved in the pathogenesis of psoriatic arthritis. Increased levels of cells that produce IL-17 are found in the circulation, joints, and skin plaques of patients with psoriatic arthritis (12).

Finally, some data seem to suggest that adipocytokines could also act by interfering with IL-17 axis $(3,13,14)$. For these reasons we decided to evaluate the possible interferences of secukinumab on different adipocytokines.

\section{MATERIALS AND METHODS}

\section{Study design}

This is a prospective study conducted in our outpatient clinic in the Rheumatology Division of Azienda Ospedaliera Universitaria Integrata di Verona. The study sample included 28 patients with PsA classified with the CASPAR criteria, recruited at our center. The study was conducted in accor- dance with the ethics principles of the Declaration of Helsinki and was approved by the local ethics committee; all the participants provided written informed consent for their participation in the study.

\section{Subjects}

The criteria for inclusion were: stable treatment with parenteral methotrexate in the last three months (ranging from $10 \mathrm{mg}$ to $15 \mathrm{mg}$ weekly), time since the diagnosis $\leq 3$ years.

The exclusion criteria for the samples of PsA patients were: systemic inflammatory diseases, active infections, neoplasms, renal, liver, endocrine or metabolic bone diseases, pregnancy and current use of biological treatment and corticosteroids. All patients received Vitamin D3 supplementation during the course of the study (7500 Ui/week).

Metabolic syndrome (MS) was diagnosed in 12 out of 28 patients in the sample (43\%), with no differences in its prevalence between men $(4 / 10,40 \%)$ and women (8/18, $44 \%$ ). The diagnosis of MS was based on the current criteria (15). These 12 patients were in treatment either for hypertension, for their dyslipidemia, or for type 2 diabetes, but their treatment did not change during the period of follow up.

The 28 joints Disease Activity Score (DAS28) was recorded at every time point. Serum samples of the patients treated with secukinumab (with doses ranging from 75 to $150 \mathrm{mg}$ every month) were stored during the treatment at baseline and then at the first, the third and the sixth month of therapy.

\section{Biochemical assessment}

Blood samples were collected and stored at $-50^{\circ} \mathrm{C}$ and later tested for adipokines. All the samples were processed in the laboratory of the Rheumatology Unit of Azienda Ospedaliera Universitaria Integrata di Verona. An ELISA assay was used for the quantitative determination of C-reactive protein (CRP) (CRP HS ELISA, DRG Instruments $\mathrm{GmbH}$, Germany), with an inter-assay $\mathrm{CV}<4.1 \%$ and an intra-assay $\mathrm{CV}<7.5 \%$. Serum concentration of human 
Table I - Demographics of the sample and values of the dosed markers at baseline (units in brackets).

\begin{tabular}{|l|c|}
\hline & Mean \pm SD \\
\hline Age & $57.39 \pm 9.94$ \\
\hline Weight $(\mathrm{kg})$ & $77.67 \pm 14.9$ \\
\hline Height $(\mathrm{cm})$ & $163.21 \pm 8.24$ \\
\hline BMI & $29.11 \pm 5.3$ \\
\hline M/F & $10 / 18$ \\
\hline CRP $\mathrm{mg} / \mathrm{L}$ & $11.9 \pm 22.51$ \\
\hline Chemerin $\mathrm{ng} / \mathrm{mL}$ & $204.9 \pm 37.79$ \\
\hline Adiponectin $\mathrm{ug} / \mathrm{mL}$ & $15.9 \pm 9.71$ \\
\hline Resistin $\mathrm{ng} / \mathrm{mL}$ & $7.84 \pm 3.179$ \\
\hline
\end{tabular}

resistin, chemerin and adiponectin were dosed using commercial ELISA kits (Mediagnost, Reutlingen, Germany). Resistin intra-assay variation was $<5 \%$ and inter-assay variation was $<6.8 \%$ with a sensitivity of $0.006 \mathrm{ng} / \mathrm{mL}$. The inter- and intra-assay $\mathrm{CV}$ of chemerin were $<5.16$ and $<2.17 \%$ respectively while the inter-assay and intra-assay $\mathrm{CV}$ of adiponectin were $<6.7 \%$ and $<4.7 \%$ respectively. The anthropometric values and adipokines' concentrations are shown in Table I.

\section{Statistical analysis}

All statistical analyses were performed per protocol by SPSS software, Version 22 (SPSS, Inc., Chicago, IL, USA). The differences between the values of the markers at month 1, 3 and $6 v s$ baseline were analyzed by one-sample $t$ test. Correlations were calculated using linear regression. Two sided $\mathrm{p}$ values of 0.05 or less were considered significant. Data are presented as mean $\pm \mathrm{SD}$.

\section{RESULTS}

The demographics of the sample and the values of the dosed markers at baseline are shown in Table I. The percentage changes from baseline to month 1 (M1), month 3 (M3) and month 6 (M6) of PCR, resistin, adiponectin and chemerin are shown in Table II as mean \pm standard deviation (SD). The weight of the patients, the levels of CRP and the values of DAS28 did not show a significant change at any point (data not shown).

We transformed CPR values into LnCRP to obtain a normal distribution for this parameter.

Table II - Percentage variation ( \pm SD) from baseline to the first, third and sixth month of observation of the dosed markers. DAS28 ( \pm $\mathrm{SD})$ is reported as absolute value.

\begin{tabular}{|l|c|c|c|}
\hline & Baseline to month 1 & Baseline to month 3 & Baseline to month 6 \\
\hline Resistin variation \% & $2.75 \pm 17.45$ & $-4.91 \pm 19.44$ & $-1.19 \pm 21.85$ \\
\hline Adiponectin variation \% & $-3.61 \pm 15.36$ & $1.33 \pm 26.31$ & $-1.65 \pm 21.73$ \\
\hline Chemerin variation \% & $-0.81 \pm 12.78$ & $-3.52 \pm 14.82$ & $-4.50 \pm 14.79$ \\
\hline DAS28 & $3.78 \pm 1.21$ & $3.57 \pm 1.58$ & $3.41 \pm 1.55$ \\
\hline
\end{tabular}

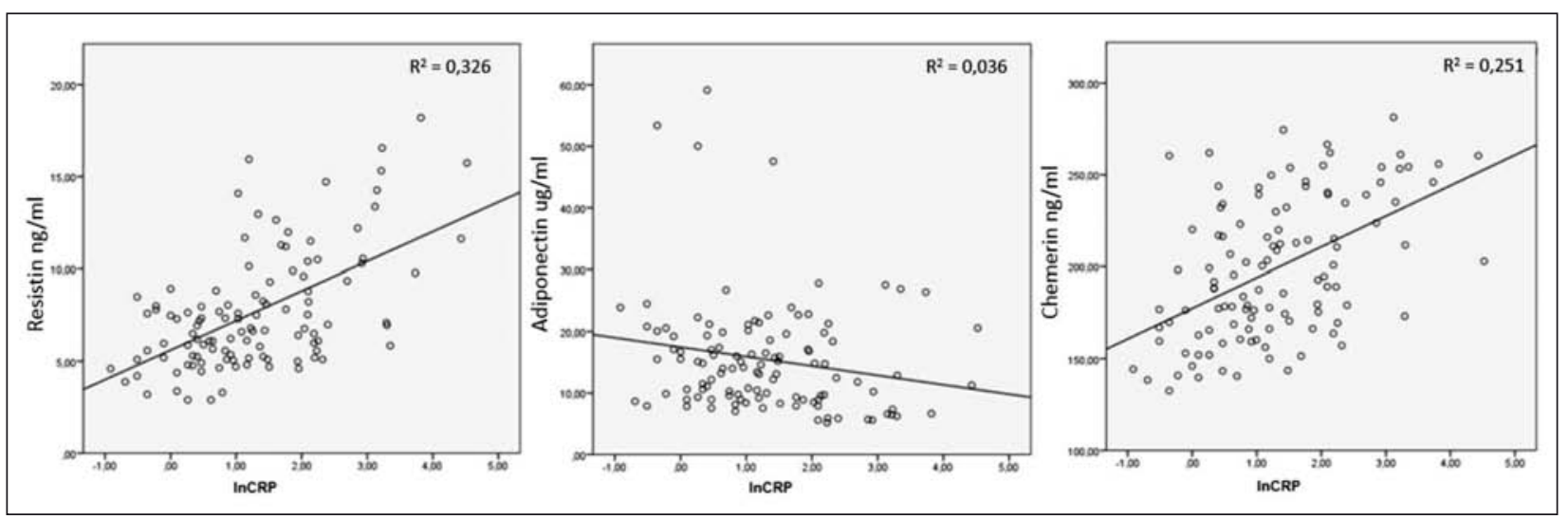

Figure 1 - Correlation between all the values of InCRP vs resistin, adiponectin and chemerin. All $p<0.05$. 
Table III - Percentage variation in males and in females, from baseline to the sixth month.

\begin{tabular}{|l|c|c|}
\hline \multicolumn{2}{|c|}{ Males $\mathrm{N}=10$} \\
\hline Weight \% variation & Baseline to month 6 & $\mathbf{p}$ \\
\hline CRP \% variation & $0.23 \pm 0.35$ & $\mathrm{Ns}$ \\
\hline Resistin \% variation & $-16.53 \pm 87.65$ & $\mathrm{Ns}$ \\
\hline Adiponectin \% variation & $-12.45 \pm 13.39$ & 0.016 \\
\hline Chemerin \% variation & $-3.00 \pm 21.28$ & $\mathrm{Ns}$ \\
\hline & $-23.81 \pm 28.76$ & 0.028 \\
\hline Weight \% variation & Females $\mathrm{N}=18$ & $\mathrm{Ns}$ \\
\hline CRP \% variation & $0.89 \pm 1.2$ & $\mathrm{Ns}$ \\
\hline Resistin \% variation & $81.05 \pm 236.50$ & Ns \\
\hline Adiponectin \% variation & $5.06 \pm 23.34$ & Ns \\
\hline Chemerin \% variation & $-0.90 \pm 22.60$ & Ns \\
\hline
\end{tabular}

A positive correlation was found between the overall values of CRP and both resistin $\left(\mathrm{p}<0.001, \mathrm{R}^{2}=0.326\right)$ and chemerin $\left(\mathrm{p}<0.001, \mathrm{R}^{2}=0.251\right)$ and a weak negative correlation for adiponectin $(\mathrm{p}<0.046$, $\mathrm{R}^{2}=0.036$ ) (Figure 1).

None of the adipokines-tested patients demonstrated a significant fluctuation when the values were tested globally, but when the values of the males were tested separately, both resistin and chemerin at M6 showed a significant decrease from baseline, while adiponectin and CRP still did not exhibit any significant variation (Table III).

No significant change was found when the females only were tested. No significant differences at baseline were observed concerning the levels of the tested adipokines between males and females.

\section{DISCUSSION}

Our data confirm both the already known relationship between proinflammatory adipokines such as resistin and chemerin and CRP (16-18), and also the expected negative correlation between adiponectin and CRP, already well described in previous studies both in non-arthritic patients (16) and in PsA patients (19).

The changes of adipokines levels during treatment with TNFi in chronic inflam- matory conditions has already been studied. When the changes in proinflammatory adipokines (such as resistin and chemerin) were considered, the results were not univocal (20-22). Also for adiponectin (a cytokine with antinflammatory action), the effects of TNFi treatment was not consistent (23-25).

This is the first time that adipokines have been evaluated during the IL-17 blockade. These are preliminary data throughout the first six months of therapy.

During this time period, the levels of adipokines showed overall no significant variation. Furthermore, when we considered the male subgroup, we found a relevant and significant decrease in the levels of both chemerin (about -12\%) and resistin (about $-24 \%$ ).

These are pro-inflammatory molecules and this remark could be of clinical relevance in patients with a chronic inflammatory disease such as PsA, despite no significant changes having been documented on CRP. Indeed, we should remember that in PsA, CRP alone is not always correlated with disease activity (26). Finally, the reduction of chemerin and resistin might exert positive effects also towards the metabolic profile of the patients. A valid explanation, possibly helpful in explaining why this finding is being observed only in the male subgroup, might be the lack of statistical power due to the limited number of subjects involved in this study (10 males and 18 females respectively). Anyway, we cannot rule out the possibility that a different gender-based response to the treatment may be present. Indeed, the correlation was lost not only when we considered the female subgroup but also when the whole study population was examined. Further studies with higher number of patients and longer times of observation are needed to define this question better.

In addition, in a murine model (27), it has been demonstrated that adiponectin may promote Th-17 cell differentiation through the upregulation of the expression of retinoid-related orphan nuclear receptor- $\gamma \mathrm{t}$ (ROR- $\gamma \mathrm{t}$ ), IL-22 and IL-23. Thus, in future 
studies, the evaluation of IL-17 concentrations along with adipokines in patients affected by PsA (and the search for any modifications during IL-17 blockade) might provide new important insights on the possible role of anti IL-17 drugs, also for the treatment of the metabolic consequences of the psoriatic disease.

\section{CONCLUSIONS}

Our study has several limitations. First, there is no control group and the sample size is limited. Second, the time extent of the observation is limited and, possibly, too short to document significant variations on some of the markers tested. Third, the traditional metabolic lab tests were not evaluated (i.e., blood glucose, HbA1c, lipid profile).

Our intent was purely to investigate whether secukinumab has any acute effect on weight and/or adipokines. These preliminary data may bring new insights for further studies concerning the possibility of specific and unexpected effects that secukinumab might have towards the adipokines profile in males and females.

Acknowledgements: the authors would like to thank the LURM (Laboratorio Universitario di Ricerca Medica) Research Center, University of Verona, especially Caterina Fraccarollo, for performing the biochemical analyses.

Conflict of interest: Dr. Idolazzi reports personal fees from Novartis and personal fees from Eli Lilly, during the conduct of the study; Dr. Gisondi reports personal fees from Abbott, Celgene, Janssen, Leopharma, Lilly, Merck Sharp \& Dohme, Novartis and Pfizer, outside the submitted work; Dr. Girolomoni reports personal fees from AbbVie, Almirall, Amgen, Boeringher-Ingelheim, Celgene, Dompè, Galderma, GlaxoSmithKline, Eli-Lilly, Hospira, Janssen, Leo Pharma, Merck Serono, Merck Sharp \& Dohme, Mundipharma, Novartis, Otsuka, Pfizer and Shiseido, outside the submitted work. All the other authors declare no conflict of interest.

\section{REFERENCES}

1. Boehncke W-H, Menter A. Burden of disease: psoriasis and psoriatic arthritis. Am J Clin Dermatol. 2013; 14: 377-88.

2. Toussirot E, Aubin F, Dumoulin G. Relationships between adipose tissue and psoriasis, with or without arthritis. Front Immunol. 2014; $5: 368$

3. Shibata S, Tada Y, Hau CS, et al. Adiponectin regulates psoriasiform skin inflammation by suppressing IL-17 production from $\gamma \delta$-T cells. Nat Commun. 2015; 6: 7687.

4. Hotamisligil GS. Inflammation and metabolic disorders. Nature. 2006; 444: 860-7.

5. Gualillo O, González-Juanatey JR, Lago F. The emerging role of adipokines as mediators of cardiovascular function: physiologic and clinical perspectives. Trends Cardiovasc Med. 2007; 17: 275-83.

6. Mariani F, Roncucci L. Chemerin/chemR23 axis in inflammation onset and resolution. Inflamm Res Off J Eur Histamine Res Soc Al. 2015; 64: 85-95.

7. Tilg H, Moschen AR. Adipocytokines: mediators linking adipose tissue, inflammation and immunity. Nat Rev Immunol. 2006; 6: 772-83.

8. Esteve E, Ricart W, Fernández-Real JM. Adipocytokines and insulin resistance. Diabetes Care. 2009; 32: S362-7.

9. Russolillo A, Iervolino S, Peluso R, et al. Obesity and psoriatic arthritis: from pathogenesis to clinical outcome and management. Rheumatol Oxf Engl. 2013; 52: 62-7.

10. Mease P. Psoriatic arthritis and spondyloarthritis assessment and management update. Curr Opin Rheumatol. 2013; 25: 287-96.

11. Mease PJ, McInnes IB, Kirkham B, et al. Secukinumab Inhibition of Interleukin-17A in patients with psoriatic arthritis. N Engl J Med. 2015; 373: 1329-39.

12. Jandus C, Bioley G, Rivals J-P, et al. Increased numbers of circulating polyfunctional Th17 memory cells in patients with seronegative spondylarthritides. Arthritis Rheum. 2008; 58: 2307-17.

13. Piccio L, Cantoni C, Henderson JG, et al. Lack of adiponectin leads to increased lymphocyte activation and increased disease severity in a mouse model of multiple sclerosis. Eur J Immunol. 2013; 43: 2089-100.

14. Kasahara DI, Kim HY, Williams AS, et al. Pulmonary inflammation induced by subacute ozone is augmented in adiponectin-deficient mice: role of IL-17A. J Immunol Baltim Md 1950. 2012; 188: 4558-67.

15. Alberti KGMM, Zimmet P, Shaw J, IDF Epidemiology Task Force Consensus Group. The metabolic syndrome--a new worldwide definition. Lancet Lond Engl. 2005; 366: 1059-62.

16. Ouchi N, Parker JL, Lugus JJ, Walsh K. Adi- 
pokines in inflammation and metabolic disease. Nat Rev Immunol 2011; 11: 85-97.

17. Bokarewa M, Nagaev I, Dahlberg L, et al. Resistin, an adipokine with potent proinflammatory properties. J Immunol Baltim Md 1950. 2005; 174: 5789-95.

18. Ernst MC, Sinal CJ. Chemerin: at the crossroads of inflammation and obesity. Trends Endocrinol Metab TEM. 2010; 21:660-7.

19. Dikbas O, Tosun M, Bes C, et al. Serum levels of visfatin, resistin and adiponectin in patients with psoriatic arthritis and associations with disease severity. Int J Rheum Dis. 2014 [Epub ahead of print].

20. Gisondi P, Lora V, Bonauguri C, et al. Serum chemerin is increased in patients with chronic plaque psoriasis and normalizes following treatment with infliximab. $\mathrm{Br} \mathrm{J}$ Dermatol. 2013; 168: 749-55.

21. Lora V, Bonaguri C, Gisondi P, et al. Autoantibody induction and adipokine levels in patients with psoriasis treated with infliximab. Immunol Res. 2013; 56: 382-9.

22. Pina T, Genre F, Lopez-Mejias R, et al. Relationship of leptin with adiposity and inflammation and resistin with disease severity in psoriatic patients undergoing anti-TNF-al- pha therapy. J Eur Acad Dermatol Venereol JEADV. 2015; 29: 1995-2001.

23. Härle P, Sarzi-Puttini P, Cutolo M, Straub RH. No change of serum levels of leptin and adiponectin during anti-tumour necrosis factor antibody treatment with adalimumab in patients with rheumatoid arthritis. Ann Rheum Dis. 2006; 65: 970-1.

24. Popa C, Netea MG, de Graaf J, et al. Circulating leptin and adiponectin concentrations during tumor necrosis factor blockade in patients with active rheumatoid arthritis. J Rheumatol. 2009; 36: 724-30.

25. Serelis J, Kontogianni MD, Katsiougiannis S, et al. Effect of anti-TNF treatment on body composition and serum adiponectin levels of women with rheumatoid arthritis. Clin Rheumatol. 2008; 27: 795-7.

26. Schoels MM, Aletaha D, Alasti F, Smolen JS. Disease activity in psoriatic arthritis (PsA): defining remission and treatment success using the DAPSA score. Ann Rheum Dis. 2016; 75: 811-8.

27. Sun X, Feng X, Tan W, et al. Adiponectin exacerbates collagen-induced arthritis via enhancing Th17 response and prompting RANKL expression. Sci Rep. 2015 [Epub ahead of print]. 